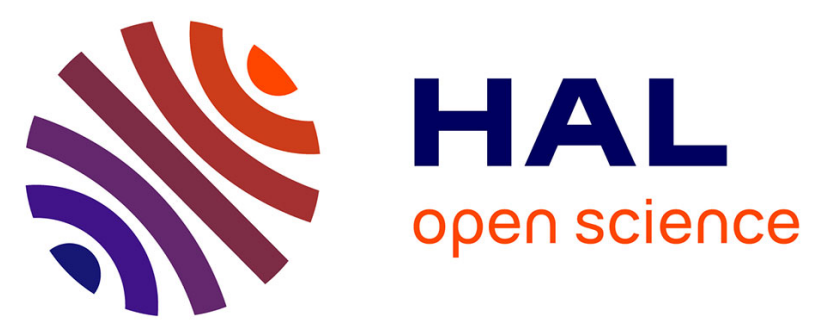

\title{
Interplay Between Microwave Spectroscopy and X-ray Diffraction: The Molecular Structure and Large Amplitude Motions of 2,3-Dimethylanisole
}

\author{
Lynn Ferres, Khai-nghi Truong, Wolfgang Stahl, Ha Vinh Lam Nguyen
}

\section{- To cite this version:}

Lynn Ferres, Khai-nghi Truong, Wolfgang Stahl, Ha Vinh Lam Nguyen. Interplay Between Microwave Spectroscopy and X-ray Diffraction: The Molecular Structure and Large Amplitude Motions of 2,3Dimethylanisole. ChemPhysChem, 2018, 19 (14), pp.1781-1788. 10.1002/cphc.201800115 . hal03183069

\author{
HAL Id: hal-03183069 \\ https://hal.science/hal-03183069
}

Submitted on 26 Mar 2021

HAL is a multi-disciplinary open access archive for the deposit and dissemination of scientific research documents, whether they are published or not. The documents may come from teaching and research institutions in France or abroad, or from public or private research centers.
L'archive ouverte pluridisciplinaire HAL, est destinée au dépôt et à la diffusion de documents scientifiques de niveau recherche, publiés ou non, émanant des établissements d'enseignement et de recherche français ou étrangers, des laboratoires publics ou privés. 


\title{
Interplay between Microwave Spectroscopy and X-ray Diffraction: The Molecular Structure and Large Amplitude Motions of 2,3- Dimethylanisole
}

\author{
Lynn Ferres ${ }^{a}$, Khai-Nghi Truong ${ }^{b}$, Wolfgang Stahla ${ }^{a}$, Ha Vinh Lam Nguyen ${ }^{*}{ }^{\circ}$
}

\begin{abstract}
To determine the structural properties of 2,3-dimethylanisole, a multidisciplinary approach was carried out where gas phase rotational spectroscopy recorded from 2 to $26.5 \mathrm{GHz}$ using a pulsed molecular jet Fourier transform microwave spectrometer was combined with solid state X-ray diffraction. Both methods revealed that only one conformer with a heavy-atom planar structure exists. In the solid state, the packing in the monoclinic space group is $P 2_{1} / n$ with $Z=4$. In the gas phase spectrum, torsional splittings due to the internal rotations of two methyl groups attached on the phenyl ring were resolved and analyzed, providing an estimate of the barriers to methyl internal rotation of $V_{3}=26.9047(5)$ and $518.7(1.2) \mathrm{cm}^{-1}$ for the methyl groups at the ortho- and meta-positions, respectively. The coupling between the two internal rotations is modeled on a two-dimensional potential energy surface, which was mapped out by quantum chemical calculations at the B3LYP/6-311++G(d,p) level of theory.
\end{abstract}

\section{Introduction}

The knowledge of the exact molecular structures of compounds is indispensable in many research fields, especially in chemistry. Since decades, this is proven by the fact that many analysis methods have been developed to determine the threedimensional structure of a molecule. Among them, diffraction represents one of the most straightforward techniques for the precise determination of molecular geometries in the solid state. In the gas phase, molecular jet Fourier transform microwave (FTMW) spectroscopy is the most suitable tool for this purpose. This work presents a multidisciplinary approach to determine the structural properties of 2,3-dimethylanisole (23DMA, also known as 1-methoxy-2,3-dimethylbenzene or 3-methoxy-o-xylene) in both, the gas phase and the solid state. This combination is ideally suited to determine the structures of sizeable molecules as only quantum chemical calculations are often insufficient..$^{[1,2]}$

[a] Lynn Ferres M. Sc., Prof. Dr. Wolfgang Stahl Institute of Physical Chemistry, RWTH Aachen University, Landoltweg 2, D-52074 Aachen, Germany

[b] Khai-Nghi Truong M. Sc. Institute of Inorganic Chemistry, RWTH Aachen University Landoltweg 1, D-52074 Aachen, Germany

${ }^{*}[\mathrm{c}]$ Dr. Ha Vinh Lam Nguyen

Laboratoire Interuniversitaire des Systèmes Atmosphériques (LISA), CNRS UMR 7583, Université Paris-Est Créteil, Université Paris Diderot, Institut Pierre Simon Laplace, 61 avenue du Général de Gaulle, F-94010 Créteil cedex, France

E-mail: lam.nguyen@lisa.u-pec.fr

Supporting information for this article is given via a link at the end of the document.
The commercially available substance 23DMA is the only isomer of the dimethylanisoles which solidifies at room temperature and, thus, eases the X-ray diffraction measurements. While the solid state structures are in general rather rigid, the results from recent gas phase investigations on o-methyl anisole $(\mathrm{OMA})^{[3]}$ and $m$-methyl anisole (MMA ${ }^{[4]}$ suggest that splittings resulting from the internal rotations of the two methyl groups attached to the phenyl ring (called the $o$ - and $m$-methyl groups from now on) can be resolved, whereas the effect arising from the methoxy methyl group is negligible. Only a very limited number of microwave spectroscopic investigations on similar aromatic system with two internal rotors are reported in the literature, e.g. 2,5-dimethylfurane, ${ }^{[5]} 2,5$-dimethylthiophene, ${ }^{[6]}$ 2-acetyl-5-methylfuran, ${ }^{[7]}$ and dimethylbenzaldehyde. ${ }^{[8]}$ The barrier heights of such large amplitude motions (LAMs) are mainly determined by steric and electronic effects. Since the two methyl groups are close to each other, we expect steric influence that causes considerable coupling between the LAMs. The electronic contribution can arise from quite distant sources in the molecule, especially when conjugated double bonds and/or aromatic systems are involved, which is also the case of 23DMA. Predicting torsional barriers is challenging because chemical intuition often fails and quantum chemical calculations are still rather inaccurate. Microwave spectroscopy yields highly accurate torsional barriers and could serve as benchmarks to improve theoretical models.

\section{Quantum Chemical Calculations}

\subsection{Conformational analysis}

Since the rotations of the three methyl groups in 23DMA do not create further conformations, the conformational landscape is completely defined by rotating the entire $-\mathrm{OCH}_{3}$ group about the $\mathrm{C}_{1}-\mathrm{O}_{14}$ bond (for atom numbering see inset in Figure 1). A potential curve was calculated, where the corresponding dihedral angle $\delta=\angle\left(\mathrm{C}_{6}-\mathrm{C}_{1}-\mathrm{O}_{14}-\mathrm{C}_{15}\right)$ was varied in a grid of $10^{\circ}$, while all other geometry parameters were optimized. Calculations were carried out at the B3LYP/6-311++G(d,p) level of theory using the Gaussian09 program package. ${ }^{[9]}$ In almost all of our recent investigations, the MP2/6-311++G(d,p) level was applied. ${ }^{[10-12]}$ However, for some previous molecules containing aromatic rings such as $\mathrm{OMA},{ }^{[3]}$ 2,5-dimethylthiophene, ${ }^{[6]}$ and phenetole, ${ }^{[13]}$ harmonic frequency calculations yielded one imaginary vibrational mode, which is a bending vibration of the phenyl ring. It is well-known that the MP2/6-311++G(d,p) level of theory often yields imaginary frequency for stable planar ring systems, which has been reported for benzene and arenes. ${ }^{[14]}$ This effect did not occur in calculations performed with the B3LYP method. Variation of the methods and basis sets in our previous studies on $\mathrm{OMA}^{[3]}$ and $p$-methyl anisole (PMA $)^{[15]}$ confirms that the structural 
parameters do not change significantly and the rotational constants are calculated with sufficient accuracy at the B3LYP/6$311++G(d, p)$ level. Therefore, we only use the results of B3LYP for the structural optimizations of 23DMA as well as for all calculations of the conformational analysis.

The calculated energies were parameterized using a Fourier expansion based on terms with the correct symmetry of $\delta$ with the corresponding coefficients given in Table S-1 in the Supporting Information. Using these Fourier coefficients, the potential energy curve was drawn as depicted in Figure 1. The obtained potential energy curve shows only one minimum at $\delta=$ $0^{\circ}$, where the methoxy group points away from the o-methyl group Similar to the case of OMA, ${ }^{[3]}$ sterical hindrance prevents the existence of a second minimum at $\delta=180^{\circ}$ because a methyl group is present at the o-position. In $m$ - and/or $p$-substituted phenyl rings such as $\mathrm{MMA}^{,\left[{ }^{[4]}\right.} \mathrm{PMA},{ }^{[15]}$ and 3,4dimethylbenzaldehyde, ${ }^{[8]}$ both conformations at $\delta=0^{\circ}$ and $180^{\circ}$ represent energy minima.

The geometry at the minimum was fully optimized to a planar conformer depicted in the inset of Figure 1. The Cartesian coordinates are given in Table S-2 in the Supporting Information. The calculated rotational constants of $A=2208.2 \mathrm{MHz}, B=$ 1103.2 $\mathrm{MHz}$, and $C=745.9 \mathrm{MHz}$ correspond to a Ray's asymmetry parameter of $\mathrm{K}=-0.51$. The predicted dipole moment components are $\mu_{\mathrm{a}}=0.89 \mathrm{D}, \mu_{\mathrm{b}}=0.80 \mathrm{D}$, and $\mu_{\mathrm{c}}=0.00 \mathrm{D}$, leading to the expectation that only $a$ - and $b$-type, but no $c$-type transitions are observable in the microwave spectrum. Frequency calculations with no imaginary frequencies confirm the stability of the optimized conformer.

\subsection{Methyl internal rotations}

For studying the internal rotations of the methyl groups two levels of theory, MP2/6-311++G(d,p) and B3LYP/6-311++G(d,p), both come into play. As we shall see below and in section 4 on the results as well as section 5 on the discussion, the results obtained using the MP2 and B3LYP methods are not always in agreements and it is not possible to confirm by the experiments which method is correct. Therefore, it is necessary to indicate the results of both methods in order to avoid a false statement or incomplete information.

The LAMs in 23DMA are complex because of three inequivalent methyl internal rotations. For the $m$-methyl group, we found a $V_{3}$ potential of $410.04 \mathrm{~cm}^{-1}$ with no significant $V_{6}$ contributions by varying the dihedral angle $\alpha=\angle\left(\mathrm{C}_{4}, \mathrm{C}_{3}, \mathrm{C}_{10}, \mathrm{H}_{12}\right)$ in a grid of $10^{\circ}$ under full relaxation of all other geometry parameters, as illustrated in Figure 2. The Fourier coefficients of this parametrized potential curve are given in Table S-3.

For the o-methyl group, the barrier height was calculated by varying the dihedral angle $\beta=\angle\left(\mathrm{C}_{1}, \mathrm{C}_{2}, \mathrm{C}_{19}, \mathrm{H}_{21}\right)$. The results obtained from calculations at the MP2/6-311++G(d,p) and B3LYP/6-311++G(d,p) levels of theory are controversial. Using the B3LYP method, three energetic minima with no significant $V_{6}$ contributions at $\beta=0^{\circ}$ and $\pm 120^{\circ}$ were obtained, indicating that the $o^{-}$and $m$-methyl groups are staggered (see Figure 2). Furthermore, local minima exist in the maximum regions around $\beta= \pm 60^{\circ}$ and $180^{\circ}$. The results obtained with the MP2 method show $V_{6}$ contributions of about $20 \%$, yielding in double minimum potentials with local maxima at $\beta_{\max }=0^{\circ}$ and $\pm 120^{\circ}$ and minima at $\beta_{\max } \pm 30^{\circ}$ (see Figure 2). These observations are probably due to the coupling between the LAMs of the two neighboring $o$ - and $m$-methyl groups.

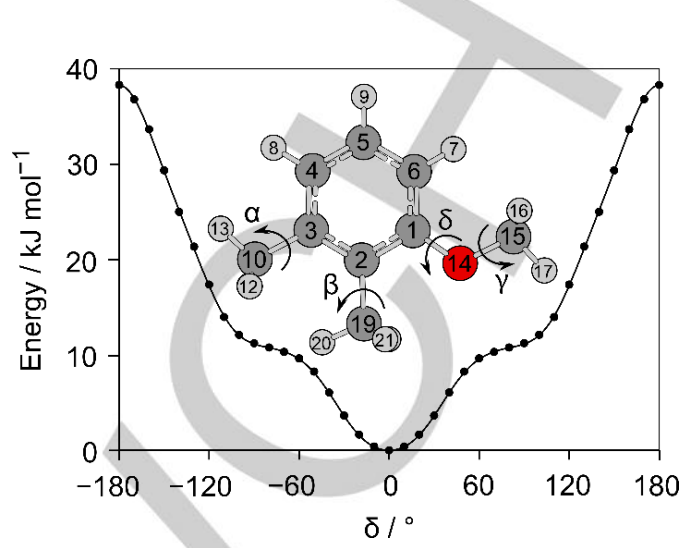

Figure 1. The potential energy curve of 23DMA obtained by rotating the methoxy group about the $\mathrm{C}_{1}-\mathrm{O}_{14}$ bond in a grid of $10^{\circ}$. The relative energies with respect to the lowest energy conformation with $E=-425.514898170$ Hartree are given. Inset: Molecular structure of the only conformer of 23DMA fully optimized at the B3LYP/6-311++G(d,p) level of theory. The protons $\mathrm{H}_{11}, \mathrm{H}_{22}$, and $\mathrm{H}_{18}$ are located behind $\mathrm{H}_{12}, \mathrm{H}_{21}$, and $\mathrm{H}_{16}$, respectively. The dihedral angles $\alpha=\angle\left(\mathrm{C}_{4}, \mathrm{C}_{3}, \mathrm{C}_{10}, \mathrm{H}_{12}\right), \quad \beta=\angle\left(\mathrm{C}_{1}, \mathrm{C}_{2}, \mathrm{C}_{19}, \mathrm{H}_{21}\right)$, and $\gamma=\angle\left(\mathrm{C}_{1}, \mathrm{O}_{14}, \mathrm{C}_{15}, \mathrm{H}_{17}\right)$ correspond to methyl internal rotations of the $m-, O^{-}$, and methoxy methyl groups about the $\mathrm{C}_{3}-\mathrm{C}_{10}, \mathrm{C}_{2}-\mathrm{C}_{19}$, and $\mathrm{O}_{14}-\mathrm{C}_{15}$ bonds, respectively.
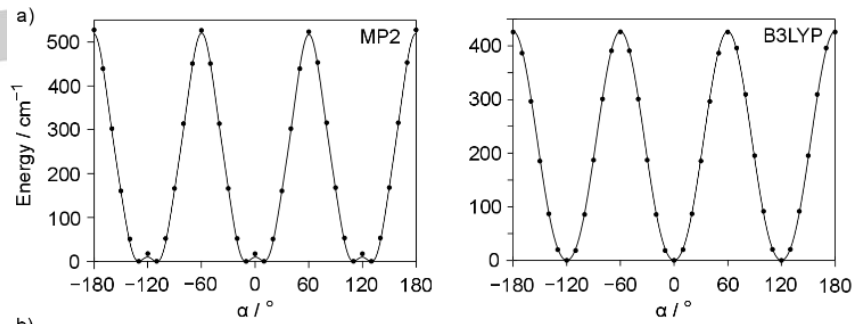

b)
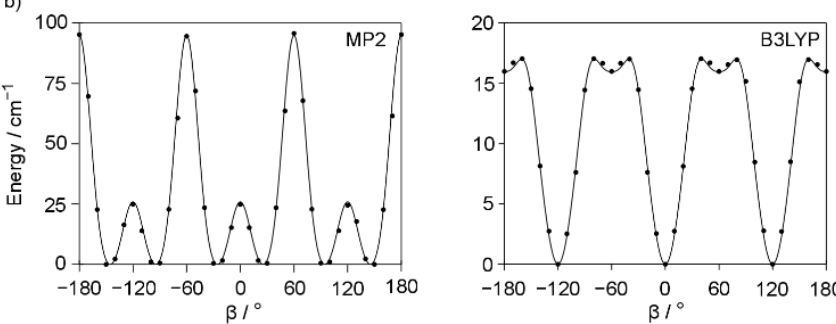

Figure 2. a) Upper trace: The potential energy curve of 23DMA obtained by rotating the $m$-methyl group about the $\mathrm{C}_{3}-\mathrm{C}_{10}$ bond by varying the dihedral angle $\alpha$ in a grid of $10^{\circ}$. The relative energies with respect to the lowest energy conformations with $E=-424.2443679$ Hartree (MP2) and $E=-425.5205477$ Hartree (B3LYP) are given. Left hand side: Double minima with small $V_{6}$ contribution at $\alpha$ around $0^{\circ}$ and $\pm 120^{\circ}$ calculated at the MP2/6-311++G(d,p) level of theory. The estimated barrier height is $527.57 \mathrm{~cm}^{-1}$. Right hand side: $V_{3}$ potential without $V_{6}$ contribution calculated at the B3LYP/6-311++G(d,p) level. The barrier height is $426.26 \mathrm{~cm}^{-1}$. b) Lower trace: The potential energy curve obtained by rotating the $o$-methyl group about the $\mathrm{C}_{2}-\mathrm{C}_{19}$ bond by varying the dihedral angle $\beta$ in a grid of $10^{\circ}$. The relative energies with respect to the lowest energy conformations with $E=-424.10748540$ Hartree (MP2) and $E=$ -425.52054770 Hartree (B3LYP) are given. Left hand side: Double minima with significant $V_{6}$ contribution (about $20 \%$ ) at $\beta$ around $\beta_{\max } \pm 30^{\circ}$ with $\beta_{\max }=0^{\circ}$ and $\pm 120^{\circ}$ calculated at the MP2/6-311++G(d,p) level of theory. The estimated barrier height is $95.76 \mathrm{~cm}^{-1}$. Right hand side: $V_{3}$ potential without $V_{6}$ contribution calculated at the $B 3 L Y P / 6-311++G(d, p)$ level. The barrier height is approximately $17.03 \mathrm{~cm}^{-1}$. 
To study this coupling, we calculated a two-dimensional potential energy surface (2D-PES) depending on the dihedral angles $\alpha$ and $\beta$. Due to symmetry, only data points in the range from $\alpha$ and $\beta=0-120^{\circ}$ are needed. The potential energies were parameterized with a 2D Fourier expansion based on terms representing the correct symmetry of the angles $\alpha$ and $\beta$. The corresponding coefficients are also available in Table S-3 in the Supporting Information. The PES indicated in Figure 3 leads to the assumption that almost no potential coupling terms between $\alpha$ and $\beta$ exist.

For the methoxy methyl group, Reinhold et al. reported a torsional barrier of about $1200 \mathrm{~cm}^{-1}$ in the case of anisole. ${ }^{[16]} \mathrm{We}$ expect a similar barrier of over $1000 \mathrm{~cm}^{-1}$ for 23DMA. Torsional splittings arising from a methyl group with such high barrier to internal rotation are not observable with our experimental resolution, which has been proven by previous investigations on monomethylanisoles. ${ }^{[3,4,15]}$

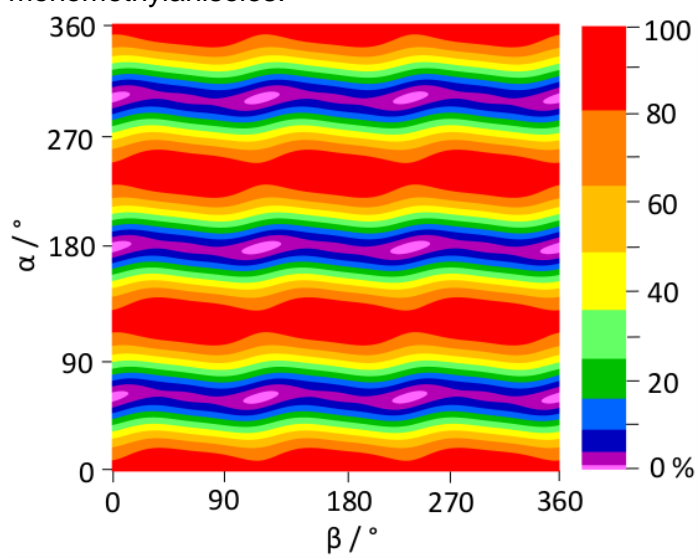

Figure 3 The potential energy surface in dependence on the dihedral angles $\alpha$ and $\beta$ of 23DMA calculated at the B3LYP/6-311++G(d,p) level of theory. The angles $\alpha$ and $\beta$ were varied in a grid of $10^{\circ}$ while all other geometry parameters were optimized. The numbers in the color code indicate the energy (in percent) relative to the energetic minimum $E_{\min }=-425.52054770$ Hartree $(0 \%)$ and the energetic maximum $E_{\max }=-425.5183019$ Hartree $(100 \%)$. Note that there are significantly more colors in the lower $50 \%$ section.

\section{Results}

\subsection{Solid state by X-ray diffraction}

The asymmetric unit contains one 23DMA molecule. Figure 4 represents the displacement ellipsoid plot of (1). Single crystal Xray diffraction experiments confirmed the structure as 23DMA. Compound (1) crystallizes in the monoclinic space group $P 2_{1} / n$ with $Z=4$. A closer look at the crystal packing gives information that no classical $\mathrm{O} \cdots \mathrm{H}$ hydrogen bonds as found in water are formed between the molecules, which is often assumed from chemical intuition for molecules like 23DMA, which have rather an "exposed" oxygen atom in its structure. The crystal structures are stabilized over the so-called $\mathrm{C}-\mathrm{H}-\pi$ interactions, which is a weak intermolecular interaction of a $\mathrm{RC}-\mathrm{H}$ group with a $\pi$ system. In the case of 23DMA, one of the hydrogens of the m-methyl group is pointing towards the $\pi($ ring) system of the neighboring 23DMA and vice versa. In conclusion, a pair of two molecules of (1) is always connected together. An overview of such a connected pair is represented in Figure 5. The distances between the hydrogen atoms and the $\pi$ (ring) system amounts 2.993(1) Á with an angle of $160.65(1)^{\circ}$ between $\mathrm{C}-\mathrm{H} \cdots \pi($ ring). Such interactions are common in crystal structures and have been reported, both in the experimental[ ${ }^{[17-19]}$ and theoretical[ ${ }^{[20,21]}$ approaches.

The crystal structure of anisole ${ }^{[22]}$ is similarly stabilized like (1). Seidel and Goddard call the interaction between the molecules edge-to-face or weak $\mathrm{C}-\mathrm{H} \cdots \pi$ interaction. The molecules appear such as in (1) only in pairs where one of the hydrogen atoms of the methoxy methyl group is pointing towards the neighboring phenyl group and vice versa.

A search in the database ${ }^{[23,24]}$ for anisole as coordination product or as co-crystal yielded 71 results. For monomethylsubstituted anisole, the number of results in the Cambridge Structural Database (CSD) reduces to one (OMA), four (MMA), and six (PMA). Higher methyl-substituted anisoles are even less commonly used as coordination product or as co-crystal; only three results have been reported for 3,4-dimethylanisole where it crystallizes as co-crystal. ${ }^{[25]}$ We report our solid as the first crystal structure for 23DMA. Crystal data, data collection parameters, and structure refinement details are summarized in Table 1.

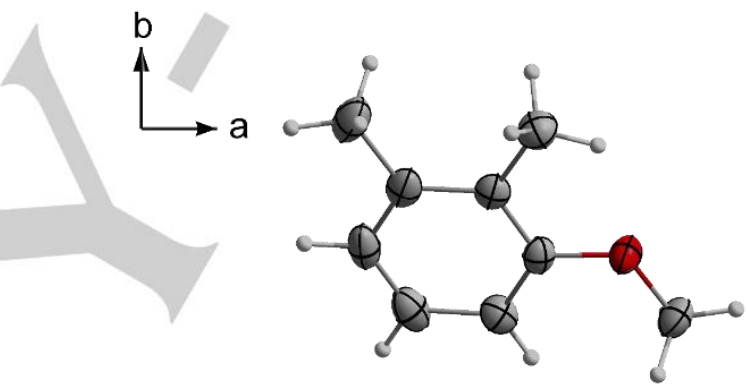

Figure 4. Displacement ellipsoid plot of the asymmetric unit in (1). The structure was drawn with a probability level of $70 \%$. The colours grey, white, and red represent carbon, hydrogen, and oxygen atoms, respectively.

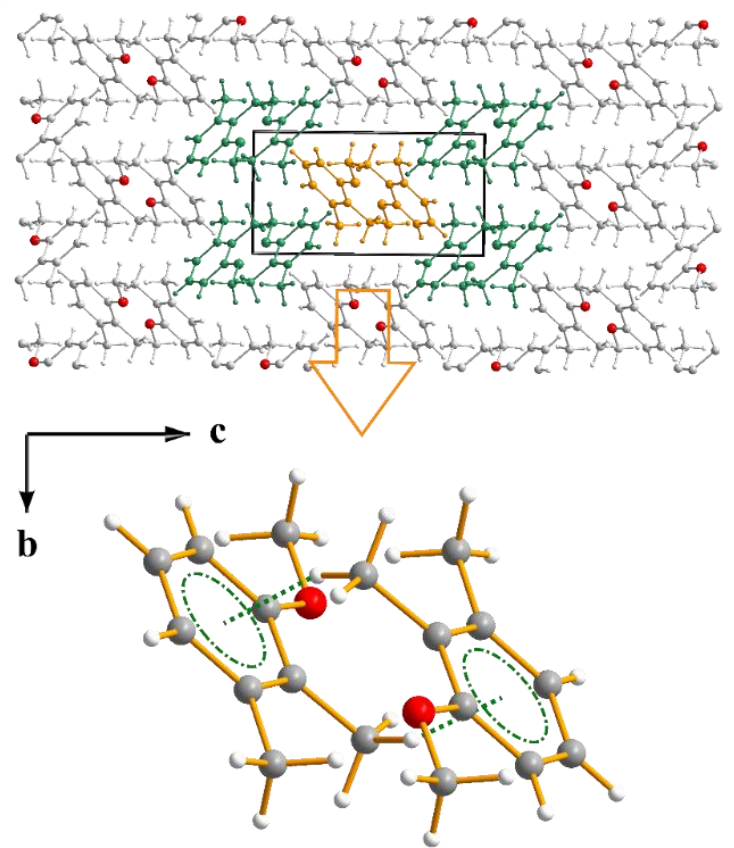

Figure 5. Upper trace: Molecular packing in the crystal packing of (1) in view direction $\left[\begin{array}{lll}1 & 0 & 0\end{array}\right]$. Lower trace: Enlarged illustration of $\mathrm{CH} \cdots \pi$ interactions between two 23DMA molecules, highlighted by green dots. 
Table 1. Crystal data and refinement results for compound (1).

\begin{tabular}{ll}
\hline Crystal data & \\
Chemical formula & $\mathrm{C}_{9} \mathrm{H}_{12} \mathrm{O}$ \\
$M_{r}$ & 136.19 \\
Crystal system, space group & Monoclinic, $P 2_{1} / n$ \\
$a, b, c(\AA)$ & $11.5063(18), 5.9621(16), 12.2176(19)$ \\
$\alpha, \beta, Y\left(^{\circ}\right)$ & $90,112.142(7), 90$ \\
$V\left(\AA^{3}\right)$ & $776.3(3)$ \\
$Z$ & 4 \\
$\mu\left(\mathrm{mm}^{-1}\right)$ & 0.074 \\
Crystal size $(\mathrm{mm})$ & $0.31 \times 0.30 \times 0.28$ \\
Crystal colour, shape & colorless block \\
& \\
Data collection & \\
Scaling and absorption correction & Multi-scan SADABS \\
$T_{\text {min }}, T_{\text {max }}$ & $0.531,0.746$ \\
No. of measured, independent and & $11286,2330,1711$ \\
observed $[I>2 \sigma(I)]$ reflections & \\
$R$ int & 0.0452 \\
$($ sin $\theta / \lambda)$ max $\left(\AA^{-1}\right)$ & 0.688 \\
& \\
Refinement & \\
$R\left[F^{2}>2 \sigma\left(F^{2}\right)\right], w R\left(F^{2}\right)$ & $0.0517,0.1383$ \\
$S$ & 1.039 \\
Number of reflections & 2330 \\
Number of parameters & 94 \\
Noumber of restraints & 0 \\
$H$-atom treatment & $H$-atom parameters constrained \\
$\Delta \rho_{\text {max }}, \Delta \rho_{\text {min }}\left(\mathrm{e} \AA^{-3}\right)$ & $0.350,-0.215$ \\
&
\end{tabular}

Experiments were carried out at $100(2) \mathrm{K}$ with Mo-K $\mathrm{K}_{\alpha}$ radiation using a CCD area detector diffractometer All C-C bonds are associated with local maxima of electron density, see checkCIF report at the end of the supplementary material. Supplementary crystallographic data can be obtained free of charge from the Cambridge Crystallographic Data Centre via http://www.ccdc.cam.ac.uk/data_request/cif (CCDC 1555874).

The rotational constants can be calculated from the X-rayextracted molecular coordinates, which are $A=2252.0952 \mathrm{MHz}$, $B=1120.0788 \mathrm{MHz}$, and $C=756.8023 \mathrm{MHz}$. The angles between the internal $o$-methyl rotor axis $i$ and the principal $a, b$, and $c$-axis are $\angle(i, a)=89.41^{\circ}, \angle(i, b)=1.09^{\circ}$, and $\angle(i, c)=89.08^{\circ}$. The respective values for the $m$-methyl rotor are $28.96^{\circ}, 61.08^{\circ}$, and $89.34^{\circ}$.

\subsection{Gas phase by microwave spectroscopy}

At the beginning, we neglected the internal rotation effects and considered 23DMA as a rigid rotor. Using the rotational constants and dipole moment components obtained from quantum chemistry (see Section 2.1) we calculated a theoretical microwave spectrum with the program $X I A M^{26]}$ in its rigid-rotor mode for a comparison with the experimental data. The $J=7 \leftarrow 6$ a-type transitions with $K_{\mathrm{a}}=0,1$ were assigned first. Afterwards, more a-type lines could be found easily. Even though quantum chemistry predicted almost the same value for dipole moment components in $a$ - and $b$-directions $\left(\mu_{\mathrm{a}}=0.89 \mathrm{D}\right.$ and $\left.\mu_{\mathrm{b}}=0.80 \mathrm{D}\right)$, $b$-type transitions appeared much weaker, but finally could be assigned and included in a fit containing in total 115 rigid-rotor lines (indicated as Fit 00 in Table 2). The standard deviation is within the measurement accuracy; the rotational and centrifugal distortion constants are determined very accurately.

Each rotational transition of 23DMA splits into five torsional components $\left(\sigma_{1} \sigma_{2}\right)=(00),(01),(10),(11)$, and (12) because of the LAMs of two methyl groups. The notations $\sigma_{1}$ and $\sigma_{2}{ }^{\left[{ }^{[6]}\right.}$ refer to the methyl rotor at the $m$ - and o-position, respectively. Figure 6 illustrates a typical spectrum. In the second step, we considered the internal rotation of the $m$-methyl rotor and predicted a one-top spectrum using the rotational constants from the rigid rotor fit and the $V_{3}$ potential as well as the angle between the internal rotor axis and the principal a-axis from quantum chemistry. Because of the strong correlation between $V_{3}$ and the moment of inertia of the methyl group $F_{0}$, the latter was fixed to $158 \mathrm{GHz}$, corresponding to $I_{\alpha}=3.2 u \AA^{2}$, a value frequently found for methyl groups. The LAM splittings between the $(00)$ and (10) species are in most transitions smaller than $1 \mathrm{MHz}$, which simplified the assignment. At this stage, we were able to fit 232 lines with $J \leq 13$ and $K_{a} \leq 5$ to a standard deviation within the measurement accuracy using the program XIAM. The results are given as Fit 00/10 in Table 2 .

In the third step, we attempted to assign the torsional species (01) arising from the o-methyl rotor with the same method previously applied for the $m$-methyl group. The assignments were more difficult because the barrier height is very low, causing large splittings in the microwave spectrum. We first identified the (01) species of some a-type transitions with small splittings, and gradually increased the number of (01) lines in the fit until finally, 188 lines were assigned and included in Fit 00/01 also given in Table 2. The standard deviation of $90.4 \mathrm{kHz}$ of this fit is much larger than the measurement accuracy. This observation has been found previously in other low barrier internal rotation problems treated by XIAM, such as ethyl acetate (101.606(23) $\left.\mathrm{cm}^{-1}, 85.3 \mathrm{kHz}\right),{ }^{[27]}$ allyl acetate $\left(98.093(12) \mathrm{cm}^{-1}, 54.0 \mathrm{kHz}\right),{ }^{[28]}$ vinyl acetate $\left(151.492(34) \mathrm{cm}^{-1}, 92.3 \mathrm{kHz}\right),{ }^{[29]}$ and 3-pentyn-1-ol $\left(9.4552(94) \mathrm{cm}^{-1}, 20.7 \mathrm{kHz}\right) .^{[30]}$ In our recent study on two conformers of MMA, we compared the results of the fits obtained by XIAM with those obtained by a newly developed program, aixPAM, where further parameters in addition to those fitted in $X I A M$ were included. ${ }^{[4]}$ Without these parameters, the standard deviations are similar in both cases for the same set of transitions and parameters $\left(27 \mathrm{kHz}\right.$ vs. $23 \mathrm{kHz}$ for the cis conformer with $V_{3}$ $=56 \mathrm{~cm}^{-1}$ and $32.1 \mathrm{kHz}$ vs. $28.3 \mathrm{kHz}$ for the trans conformer with $V_{3}=37 \mathrm{~cm}^{-1}$ ). With three additional parameters, the standard deviation of aixPAM decreases to $3.7 \mathrm{kHz}$ and $4.1 \mathrm{kHz}$ for the cis and the trans conformer, respectively. Because the $V_{3}$ potential of the o-methyl rotor is only about $27 \mathrm{~cm}^{-1}$, we were confident that the large standard deviation obtained from XIAM for the o-methyl group of 23DMA also arises from the limited number of fitted parameters. Due to the large standard deviation, the centrifugal distortion constants could not be fitted well and therefore were fixed to the values obtained from Fit 00/10.

At this stage, we used the program aixPAM to fit the same set of $(00)$ and (01) species. By adding 5 parameters, which are $V_{J}$ multiplying $\mathrm{P}^{2}[1-\cos (3 \alpha)], V_{K}$ multiplying $\mathrm{P}_{\mathrm{z}}^{2}[1-\cos (3 \alpha)], V_{-}$ multiplying $\left(\mathrm{P}_{\mathrm{x}}{ }^{2}-\mathrm{P}_{\mathrm{y}}{ }^{2}\right)[1-\cos (3 \alpha)]$, Pzp3 multiplying $\mathrm{P}_{\mathrm{z}} \mathrm{p}^{3}$, and D2p2 multiplying $\left(P_{x}{ }^{2}-P_{y}{ }^{2}\right) p^{2}$, we obtained a standard deviation of 3.6 $\mathrm{kHz}$, which is much closer to the measurement accuracy. This fit is given as Fit aixPAM in Table 2, and clearly confirms that the (01) species assignment is correct and the large standard deviation of Fit 00/01 arises from the lack of higher order term parameters. The added parameters are effective parameters which take into account the higher order terms of the $V_{3}$ potentials as well as the quartic higher order combinations. 
Table 2. Molecular parameters of $p$-methyl anisole in the principal axis system obtained by the XIAM and aixPAM codes.

\begin{tabular}{|c|c|c|c|c|c|c|c|}
\hline Par. $^{a}$ & Unit & Fit $00^{b}$ & $\begin{array}{l}\text { Fit } 00 / 10^{c} \\
\text { XIAM }\end{array}$ & $\begin{array}{l}\text { Fit } 00 / 01^{d} \\
\text { XIAM }\end{array}$ & $\begin{array}{l}\text { Fit } 00 / 01^{e} \\
\text { aixPAM }\end{array}$ & $\begin{array}{c}\text { Global Fit }^{\mathfrak{T}} \\
\text { XIAM }\end{array}$ & Calc. ${ }^{g}$ \\
\hline $\bar{A}$ & $\mathrm{MHz}$ & $2204.62202(26)$ & $2204.60333(50)$ & $2204.5825(63)$ & 2246.7(15) & $2204.603(13)$ & 2208.1534 \\
\hline B & $\mathrm{MHz}$ & $1114.47007(16)$ & $1114.47150(12)$ & $1107.7520(16)$ & $1103.06(40)$ & $1107.7338(26)$ & 1103.2195 \\
\hline C & $\mathrm{MHz}$ & $748.079761(88)$ & $748.077482(68)$ & 748.07934(96) & 749.02(32) & $748.0765(17)$ & 745.9185 \\
\hline$\Delta_{\lrcorner}$ & $\mathrm{kHz}$ & $0.03147(68)$ & $0.03169(45)$ & $0.03169^{h}$ & $0.03685(82)$ & $-0.072(11)$ & 0.0200599 \\
\hline$\Delta_{\mathrm{JK}}$ & $\mathrm{kHz}$ & $0.1366(43)$ & $0.1351(29)$ & $0.1351^{\mathrm{h}}$ & $0.0269(54)$ & $0.212(93)$ & 0.1010498 \\
\hline$\Delta_{\mathrm{K}}$ & $\mathrm{kHz}$ & --- & $-0.116(41)$ & $-0.116^{h}$ & $-0.176(58)$ & $3.93(95)$ & 0.0022304 \\
\hline$\delta_{\jmath}$ & $\mathrm{kHz}$ & $0.01197(36)$ & $0.01206(24)$ & $0.01206^{\mathrm{h}}$ & $0.01466(43)$ & $-0.0434(61)$ & 0.0064910 \\
\hline$\delta_{K}$ & $\mathrm{kHz}$ & $0.1428(63)$ & $0.1395(51)$ & $0.1395^{\mathrm{h}}$ & 0.0994(82) & --- & 0.0395049 \\
\hline $\mathrm{V}_{3}$ & $\mathrm{~cm}^{-1}$ & --- & $520.08(39)$ & $26.9068(7)$ & $26.756(16)$ & $18.7(1.2) / 26.9047(5)^{i}$ & 16.9653 \\
\hline $\mathrm{Dpi}_{2\lrcorner}$ & $\mathrm{MHz}$ & --- & --- & $0.18440(87)$ & $0.302(14)$ & $0.18513(66)$ & \\
\hline $\mathrm{Dpi}_{2 \mathrm{~K}}$ & $\mathrm{MHz}$ & --- & --- & $-0.3149(55)$ & $-3.234(96)$ & $-0.3125(47)$ & \\
\hline $\mathrm{Dpi}_{2-}$ & $\mathrm{MHz}$ & --- & --- & $0.2143(12)$ & $0.168(36)$ & $0.21593(90)$ & \\
\hline $\mathrm{V}_{\mathrm{J}}$ & $\mathrm{MHz}$ & --- & --- & --- & $2.31(25)$ & --- & \\
\hline $\mathrm{V}_{-}$ & $\mathrm{MHz}$ & --- & --- & --- & $3.49(40)$ & --- & \\
\hline $\mathrm{V}_{\mathrm{K}}$ & $\mathrm{GHz}$ & --- & --- & --- & $-0.0549(17)$ & --- & \\
\hline Pzp3 & $\mathrm{MHz}$ & --- & --- & --- & $-0.2797(40)$ & --- & \\
\hline D2p2 & $\mathrm{GHz}$ & --- & --- & --- & $0.472(29)$ & --- & \\
\hline$\angle(\mathrm{i}, \mathrm{a})$ & $\circ$ & --- & $23.46(52)$ & $87.79(1)$ & $89.323(21)$ & $21.8(1.1) / 87.79(1)^{i}$ & $29.42 / 86.15^{i}$ \\
\hline$\angle(\mathrm{i}, \mathrm{b})$ & $\circ$ & --- & $66.54(52)$ & $2.21(1)$ & $0.677(21)$ & $68.2(1.1) / 2.21(1)^{\mathrm{i}}$ & $60.58 / 3.85^{i}$ \\
\hline$\angle(\mathrm{i}, \mathrm{c})$ & $\circ$ & --- & $90.0^{k}$ & $90.0^{k}$ & $90.0^{k}$ & $90.0 / 90.0^{i, k}$ & $89.95 / 90.00^{i}$ \\
\hline$N^{\prime}$ & & 115 & $115 / 117$ & $115 / 73$ & $115 / 73$ & $115 / 117 / 81 / 77 / 75$ & \\
\hline$\sigma^{m}$ & $\mathrm{kHz}$ & 3.2 & 3.0 & 90.4 & 3.6 & 117.6 & \\
\hline
\end{tabular}

${ }^{a}$ All parameters refer to the principal axis system. Watson's A reduction and $I r$ representation were used. ${ }^{b}$ Rigid rotor fit including only $(00)$ species. ${ }^{c}$ Including only the $(00)$ and $(10)$ torsional species of the $m$-methyl rotor, fitted using the program XIAM. ${ }^{d}$ Including only the $(00)$ and $(01)$ torsional species of the $o$-methyl rotor, fitted using the program XIAM. ${ }^{\mathrm{d}}$ Including only the $(00)$ and $(01)$ torsional species of the $o$-methyl rotor, fitted using the program aixPAM. ${ }^{\dagger} \mathrm{Global}$ fit using the program XIAM. ${ }^{g}$ Calculated at the B3LYP/6-311++G(d,p) level of theory. ${ }^{\mathrm{h}}$ Fixed to the values obtained from Fit $00 / 10 .{ }^{i}$ Left and right hand side: values obtained for the $m$ - and $o$-methyl groups, respectively. ${ }^{k}$ Fixed due to symmetry. ${ }^{~}$ Number of lines. Fit 00: $(00)$ species, Fit 00/10: (00)/(10) species, Fit 00/01: $(00) /(01)$ species, Global Fit: $(00) /(01) /(10) /(11) /(12)$ species. ${ }^{m}$ Standard deviation of the fit.

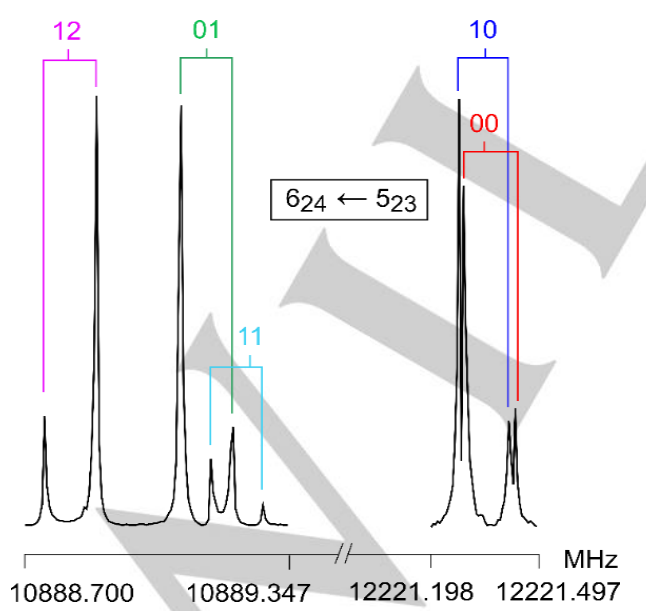

Figure 6: An exemplary a-type rotational transition (in $\mathrm{MHz}$ ) measured at high resolution, picturing typical splittings into five torsional species. The brackets describe Doppler pairs. The spectra are normalized. For the left hand side spectrum, 780 decays were co-added and the polarization frequency was $10888.85 \mathrm{MHz}$. For the right hand side spectrum, the number of co-added decays was 50 and the polarization frequency $12221.50 \mathrm{MHz}$.
Finally, we predicted a two-top spectrum using the program $X I A M$ in order to assign the (11) and (12) species, which describe the coupling between the LAMs of two methyl groups. For transitions with large torsional splittings, the assignments were evident. On the other hand, there are some transitions with splittings in the same order of the standard deviation of Fit 00/01 (a few $100 \mathrm{kHz}$ ), causing the distinction of the (11) and (12) species of those lines much more uncertain. In a global fit containing 465 lines carried out with XIAM, we observed a standard deviation of $118 \mathrm{kHz}$ by floating 14 parameters, which are the rotational constants, five quartic centrifugal distortion constants, the two $V_{3}$ potentials, angles between the internal rotor axes and the principal a-axis, and three higher order parameters $D_{\text {pi2J, }} D_{\text {pi2K, }}, D_{\text {pi2- }}$ of the o-methyl rotor. The results of this fit are summarized as Global Fit in Table 2. A list of all frequencies is available in Table S-6; a comparison between the experimental and the theoretical spectrum simulated by XIAM in Figure S-1 in the Supporting Information. 


\section{Discussion}

The rotational- and quartic centrifugal distortion constants were determined with very high accuracy by fitting 115 torsional transitions in a rigid rotor fit. The standard deviation of $3.2 \mathrm{kHz}$ is close to the measurement accuracy. The $m$-methyl group with an intermediate $V_{3}$ potential of $523.39(39) \mathrm{cm}^{-1}$ does not cause fitting problems for the program XIAM. The one-top Fit 00/10 reaches the same quality as that of the rigid rotor Fit 00 by additionally including only two parameters, namely the barrier height $V_{3}$ and the angle between the $m$-methyl rotor axis and the principal $a$-axis

In the contrary, the internal rotation of the o-methyl group cannot be captured correctly by XIAM. With a $V_{3}$ potential of only $27.0750(6) \mathrm{cm}^{-1}$, the addition of three higher-order parameters $D_{\text {pizJ, }} D_{\text {pizK, }}$ and $D_{\text {piz- }}$ were not sufficient to reduce the standard deviation to measurement accuracy. With a standard deviation of $90.4 \mathrm{kHz}$, all centrifugal distortion constants in Fit 00/01 could not be determined well.

The torsional barrier found for the o-methyl group in OMA is rather high $\left(459 \mathrm{~cm}^{-1}\right)$ and the program XIAM yielded excellent fitting results for this LAM problem. We have proposed that the sterical hindrance between the methoxy and the o-methyl group is the main reason for this intermediate value of $V_{3}$, which has been confirmed by the results obtained for a number of $O$ substituted toluenes. ${ }^{[3]}$ In MMA, the methyl group at the $m$-position is further apart from the methoxy group, which creates a symmetric local environment near the methyl group and decreases the ring methyl barrier height by an order of magnitude (36.6342(84) and 55.7693(90) $\mathrm{cm}^{-1}$ for the trans and the cis conformer, respectively). ${ }^{[4]}$ However, in 23DMA the intermediate barrier of about 523.39 (39) $\mathrm{cm}^{-1}$ of the $m$-methyl group is not surprising because the o-methyl group in the neighborhood causes sterical hindrance. The very low barrier of the o-methyl group can be explained in a similar way as has been described in Refs. ${ }^{[31]}$ and ${ }^{[32]}$. If we assume that the methoxy methyl group and the two other methyl groups are similar, then the o-methyl group, which has a $\mathrm{C}_{3 \mathrm{v}}$ symmetry, experiences potentials based on a $\mathrm{C}_{2 \mathrm{v}}$ frame symmetry as in toluene $\mathrm{CH}_{3}-\mathrm{C}_{6} \mathrm{H}_{5}\left(V_{6}=4.8 \mathrm{~cm}^{-1}\right)^{[33]}$ or nitromethane $\mathrm{CH}_{3} \mathrm{NO}_{2}\left(V_{6}=4.9 \mathrm{~cm}^{-1}\right),{ }^{[34]}$ where only a $V_{6}$ term but no $V_{3}$ contribution exists. In the case of 23DMA, the frame symmetry is slightly out-of-balance, causing the small $V_{3}$ potential term of about $29 \mathrm{~cm}^{-1}$ observed for the o-methyl group. Unfortunately, $V_{6}$ term cannot be fitted because only data in the ground state are available, i.e. our attempts to fit a combined $V_{3^{-}}$ $V_{6}$ function as suggested by the MP2 method (see section 2.2.) failed due to experimental limitation. Thus, the experiments cannot yield any statement whether MP2 or B3LYP, which states dominant $V_{3}$ terms, is better suitable to describe the internal rotations in 23DMA.

In our recent investigation on MMA, the new code aixPAM has been introduced, which provides the possibility to add effective Hamiltonian terms from the input file. ${ }^{[4]}$ This code has proven to be quite satisfactory to treat low barrier internal rotation problems. When it was applied to $183 \mathrm{~A}$ and $137 \mathrm{E}$ lines in the spectrum of trans-MMA with $J \leq 13$ and $K_{a} \leq 4$, only 17 parameters were necessary to yield a standard deviation of $4.1 \mathrm{kHz}$, close to the measurement accuracy and corresponding to 18.8 lines/parameter. Similarly, when aixPAM was applied to $92 \mathrm{~A}$ and $131 \mathrm{E}$ lines of cis-MMA, the same number of parameters were needed to generate a standard deviation of $3.7 \mathrm{kHz}$ corresponding to 13.1 lines/parameter. For the o-methyl group of 23DMA, we added in Fit 00/01 obtained by aixPAM 5 effective terms $V_{J}, V_{K}, V_{-}$, Pzp3, and D2p2 not available in Fit 00/01 obtained by XIAM. This enabled us to achieve a standard deviation close to the experimental accuracy, where similar to the cases of cis- and trans-MMA, the operators containing the $[1-\cos (3 \alpha)]$ term led to most significant changes of the fit. However, in the aixPAM fits, the rotational constants are highly correlated with the higher order parameters, especially $V_{J}, V_{K}, V_{-}$ which are not fitted in XIAM. This decreases the accuracy of the rotational constants obtained with aixPAM.

The global two-top fit obtained with XIAM including 115 (00), 117 (01), 81 (10), 77 (11), and 75 (12) lines, delivered a standard deviation of $118 \mathrm{kHz}$. Further fitting using the available parameters allowed us to reduce the residuals significantly. We believe that the currently large standard deviation is due to the limited number of fitted parameters in XIAM, which has been proven by comparing Fit 00/01 XIAM with Fit 00/01 aiXPAM. In agreement with quantum chemical calculation, no extra splittings above the $\mathrm{kHz}$ level occur from the internal rotation of the methoxy methyl group.

Structural parameters such as rotational constants and the angles between the internal rotor axes and the principal axes calculated at the B3LYP/6-311++G(d,p) level of theory agree well with the experimental values (see Table 2). Therefore, we conclude that the B3LYP/6-311++G(d,p) level is sufficiently suited for optimizing the structures of 23DMA. On the other hand, the $V_{3}$ potential of the o-methyl group obtained with the B3LYP method is closer to the experimental value than that calculated with the MP2 method. The results are inverse for the $V_{3}$ potential of the $m$ methyl group. In independence of this, the barrier height calculated by the MP2 method is closer to the experimental one for the $m$-methyl group, while for the o-methyl group, the value calculated by the B3LYP method is closer (see also Table 2).

The gas phase structure yielded from microwave spectroscopy is similar to that in the solid state deduced from $X$ ray diffraction. In agreement with quantum chemistry, only one conformer of 23DMA exists in both states of aggregation, where the methoxy methyl group is pointed away from the o-methyl group. The rotational constants of this conformer obtained in the solid state is very close to those deduced from the aixPAM Fit $00 / 01$, confirming that almost the same molecular structure is present in both states. Furthermore, the angles between the internal rotor axes and the principal axes obtained in the solid state match the calculated values well, while a small deviation of about $7^{\circ}$ was observed for the $m$-methyl rotor angles $\angle(i, a)$ and $\angle(i, b)$. We note that the planar angle $\mathrm{C}_{1} \mathrm{O}_{14} \mathrm{C}_{15}$ is nearly invariant in all calculations for the methyl internal rotations, but varies in the conformational analysis from $114.6^{\circ}$ to $125.59^{\circ}$. In the crystal, the experimental value found by $\mathrm{X}$-ray is $117.45^{\circ}$, which is very close to the optimized value of $118.80^{\circ}$.

In the rotational spectrum, only very few weak lines remained unassigned, leading to our conclusion that 23DMA dimer was not present in our jet-cooled spectrum. Changing the measurement conditions in order to obtain structural information for this dimer is an interesting outlook for a comparison with the molecular arrangement in the crystal packing illustrated in Figure 5. 


\section{Experimental section}

A broadband scan in the frequency range from 10 to $14 \mathrm{GHz}$ (illustrated in Figure S-1 in the Supporting Information) was recorded by overlapping spectra with $0.25 \mathrm{MHz}$ step size using a molecular jet FTMW spectrometer operating from 2.0 to $26.5 \mathrm{GHz} .{ }^{[35]}$ The substance with a purity of $98 \%$ was purchased from $\mathrm{TCl}$, Eschborn, Germany, and measured without further purification. A piece of a pipe cleaner was soaked in the substance and inserted in front of the nozzle. The substance was heated at approximately $70^{\circ} \mathrm{C}$ to avoid crystallization. Helium was used as carrier gas and the 23DMA-He mixture was expanded into the cavity at a backing pressure of $200 \mathrm{kPa}$. Each signal present in the broadband scan was remeasured at higher resolution and appeared as doublets due to the Doppler effect. The line width average is $30 \mathrm{kHz}$, corresponding to a measurement accuracy of $3 \mathrm{kHz}$.

Suitable crystals for single crystal $\mathrm{X}$-ray diffraction were obtained by crystallization from $\mathrm{CH}_{2} \mathrm{Cl}_{2}$ as colorless blocks. The crystals are highly temperature-sensitive and start to melt at approximately $278 \mathrm{~K}$. Intensity data were collected on a Bruker D8 goniometer with a Bruker SMART APEX CCD area detector in $\omega$-scan mode using Mo- $K_{\alpha}$ radiation $(\lambda=0.71073 \AA$ ) from an Incoatec microsource with multilayer optics. ${ }^{\left[{ }^{[6]}\right.} \mathrm{A}$ temperature of 100 (2) $\mathrm{K}$ was maintained with the help of an Oxford Cryostream 700 instrument. Data were integrated with SAINT $+;\left[{ }^{[37]}\right.$ scaling and absorption correction were based on multi-scan methods with SADABS. ${ }^{[38]}$ The structure was solved by Direct Methods using SHELXS97.[39] Full-matrix least-square refinements based on $F^{2}$ were performed with SHELXL-2013.[40,41] Non-hydrogen atoms were assigned anisotropic displacement parameters. Other hydrogen atoms were placed in idealized positions and included as riding. Isotropic displacement parameters for all hydrogen atoms were constrained to multiples of the equivalent displacement parameters of their parent atoms with $U_{\text {iso }}(H)=1.2 U_{\text {eq }}$ (parent). Five reflections with large discrepancies between calculated and observed structure factors were omitted from the least-squares refinement as outliers.

\section{Acknowledgments}

We thank A. Wildenberg for her contribution within her student's research project and the European COST initiative MOLIM for a STSM (personal grant A. Wildenberg). Prof. Dr. Ulli Englert is specially acknowledged for his tremendous support, for collecting $\mathrm{X}$-ray data, and for proof-reading the manuscript. Simulations were performed with computing resources granted by RWTH Aachen University under project <thes0248>.

Keywords: structure determination, rotational spectroscopy, Xray diffraction, internal rotation, large amplitude motion

\section{References}

[1] H. Mouhib, D. Jelisavac, W. Stahl, R. Wang, I. Kalf, U. Englert, ChemPhysChem 2011, 12, 761-764.

[2] C. Merkens, T. Stadtmüller, U. Englert, H. Mouhib, W. Stahl, Z. Naturforsch. 2014, 69a, 303-312.

[3] L. Ferres, H. Mouhib, W. Stahl, H. V. L. Nguyen, ChemPhysChem 2017, 18, 1855-1859.

[4] L. Ferres, W. Stahl, H. V. L. Nguyen, J. Chem. Phys. 2018, 148, 124304.

[5] V. Van, J. Bruckhuisen, W. Stahl, V. Ilyushin, H. V. L. Nguyen, J. Mol. Spectrosc. 2018, 343, 121-125.

[6] V. Van, W. Stahl, H. V. L. Nguyen, Phys. Chem. Chem. Phys. 2015, 17, 32111-32114.

[7] V. Van, W. Stahl, H.V.L. Nguyen, ChemPhysChem 2016, 17, 3223-3228.

[8] M. Tudorie, I. Kleiner, M. Jahn, J.-U. Grabow, M. Goubet, O. Pirali, J. Phys. Chem. A 2013, 117, 13636-13647.

[9] M. J. Frisch, G. W. Trucks, H. B. Schlegel, G. E. Scuseria, M. A. Robb, J. R. Cheeseman, G. Scalmani, V. Barone, B. Mennucci, G. A. Petersson, H. Nakatsuji, M. Caricato, X. Li, H. P. Hratchian, A. F. Izmaylov, J. Bloino, G. Zheng, J. L. Sonnenberg, M. Hada, M. Ehara, K. Toyota, R. Fukuda, J. Hasegawa, M. Ishida, T. Nakajima, Y. Honda, O. Kitao, H. Nakai, T. Vreven, J. A. Montgomery, Jr., J. E. Peralta, F. Ogliaro, M. Bearpark, J. J. Heyd, E. Brothers, K. N. Kudin, V. N. Staroverov, R. Kobayashi, J. Normand, K. Raghavachari, A. Rendell, J. C. Burant, S. S. Iyengar, J. Tomasi, M. Cossi, N. Rega, J. M. Millam, M. Klene, J. E. Knox, J. B. Cross, V. Bakken, C. Adamo, J. Jaramillo, R. Gomperts, R. E. Stratmann, O. Yazyev, A. J. Austin, R. Cammi, C. Pomelli, J. W. Ochterski, R. L. Martin, K. Morokuma, V. G. Zakrzewski, G. A. Voth, P. Salvador, J. J. Dannenberg, S. Dapprich, A. D. Daniels, O. Farkas, J. B. Foresman, J. V. Ortiz, J. Cioslowski, D. J. Fox, Gaussian 09, Revision A.02, Gaussian, Inc., Wallingford CT, 2009.

[10] R. Kannengießer, W. Stahl, H. V. L. Nguyen, I. Kleiner, J. Phys. Chem. A 2016, 120, 3992-3997.

[11] V. Van, W. Stahl, M. Schwell, H.V.L. Nguyen, J. Mol. Struct. 2018, 1156, 348-352.

[12] L. Ferres, H. Mouhib, W. Stahl, M. Schwell, H. V. L. Nguyen, J. Mol. Spectrosc. 2017, 337, 59-64.

[13] L. Ferres, W. Stahl, H. V. L. Nguyen, Mol. Phys. 2016, 114, 2788-2793.

[14] D. Moran, A. C. Simmonett, F. E. Leach, W. D. Allen, P. v. R. Schleyer, H. F. Schaefer, J. Am. Chem. Soc. 2006, 128, 9342-9343.

[15] L. Ferres, W. Stahl, I. Kleiner, H. V. L. Nguyen, J. Mol. Spectrosc. 2018, 343, 44-49.

[16] B. Reinhold, I. A. Finneran, S. T. Shipman, J. Mol. Spectrosc. 2011, 270, 89-97.

[17] M. Nishio, CrystEngComm 2004, 6, 130-158.

[18] H. Suezawa, S. Ishihara, Y. Umezawa, S. Tsuboyama, M. Nishio, Eur. J. Org. Chem. 2004, 4816-4822.

[19] J. W. Steed, J. L. Atwood, In Supramolecular Chemistry, Chichester: John Wiley \& Sons, 2009.

[20] K. Sundararajan, K. S. Viswanathan, A. D. Kulkarni, S. R. Gadre, J. Mol. Str. 2002, 613, 209-222.

[21] S. Grimme, J. Comput. Chem. 2004, 25, 1463-1473. 
[22] R. W. Seidel, R. Goddard, Acta Cryst. 2015, C71, 664-666.

[23] Cambridge Structural Database (version 5.38): CCDC: Cambridge, U.K., 2016, including updates until Nov. 2016.

[24] C. R. Groom, F. H. Allen, Angew. Chem. Int. Ed. 2014, 53, 662-671.

[25] M. J. Horner, K. T. Holman, M. D. Ward, J. Am. Chem. Soc. 2007, 129, 14640-14660.

[26] H. Hartwig and H. Dreizler, Z. Naturforsch. 1996, 51a, 923932.

[27] D. Jelisavac, D. C. Cortés-Gómez, H. V. L. Nguyen, L. W. Sutikdja, W. Stahl, I. Kleiner, J. Mol. Spectrosc. 2009, 257, 111-115.

[28] H. V. L. Nguyen, H. Mouhib, W. Stahl, I. Kleiner, Mol. Phys. 2010, 108, 763-770.

[29] H. V. L. Nguyen, A. Jabri, V. Van, W. Stahl, J. Phys. Chem. A 2014, 118, 12130-12136.

[30] K. Eibl, R. Kannengießer, W. Stahl, H. V. L. Nguyen, I. Kleiner, Mol. Phys. 2016, 114, 3483-3489.

[31] H. Mouhib, D. Jelisavac, L. W. Sutikdja, E. Isaak, W. Stahl, J. Phys. Chem. A 2011, 115, 118-122.

[32] A. Jabri, V. Van, H. V. L. Nguyen, W. Stahl, I. Kleiner, ChemPhysChem 2016, 17, 17, 2660-2665.

[33] V. V. Ilyushin, Z. Kisiel, L. Pszczółkowski, H. Mäder, J. T. Hougen, J. Mol. Spectrosc. 2010, 259, 26-38.

[34] G. O. Sørensen, T. Pedersen, H. Dreizler, A. Guarnieri, A. P. Cox, J. Mol. Struct. 1983, 97, 77-82.

[35] J.-U. Grabow, W. Stahl, H. Dreizler, Rev. Sci. Instrum. 1996, 67, 4072-4084.

[36] SMART (version 5.624): Program for Bruker CCD X-ray Diffractometer Control, Bruker AXS Inc., Madison, Wisconsin, USA, 2001.

[37] SAINT+ (version 7.68): Program for Reduction of Data Collected on Bruker CCD Area Detector Diffractometer, Bruker AXS Inc., Madison, Wisconsin, USA, 2009.

[38] SADABS: Program for Empirical Absorption Correction of Area Detector Data, Bruker AXS Inc., Madison, Wisconsin, USA, 2008.

[39] G. M. Sheldrick, Acta Cryst. 2008, A64, 112-122.

[40] G. M. Sheldrick, SHELXL13, University of Göttingen, Germany, 2013.

[41] G. M. Sheldrick, Acta Cryst. 2015, C71, 3-8. 


\section{Entry for the Table of Contents}

\section{ARTICLE}

The structure and dynamics of 2,3dimethylanisole: How good is the agreement of the gas phase and the solid state?

Accurate structural parameters were determined by microwave spectroscopy for the gas phase and Xray diffraction for the solid state with supports from quantum chemistry. Internal rotation effects arising from the $o$ - and $m$-methyl groups yielded important information on the internal dynamics of the molecule.

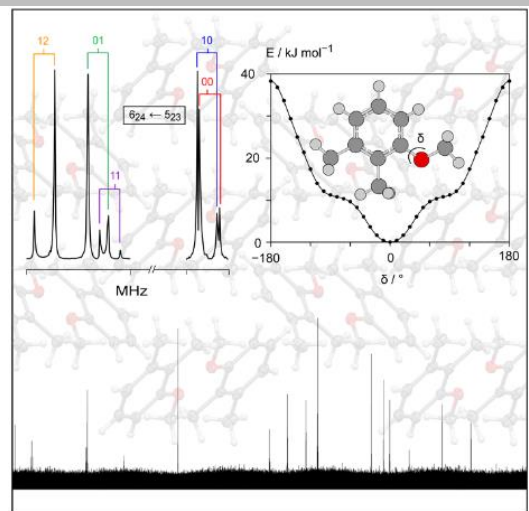

L. Ferres, K.-N. Truong, W. Stahl, H. V. L. Nguyen, ${ }^{*}$

Page No. - Page No.

Interplay between Microwave Spectroscopy and X-ray Diffraction: The Molecular Structure and Large Amplitude Motions of 2,3Dimethylanisole 